\title{
Diagnóstico de Fibrilação Atrial na Comunidade Utilizando Eletrocardiograma e Autorrelato: Análise Transversal do ELSA-Brasil
}

\author{
Atrial Fibrillation Diagnosis using ECG Records and Self-Report in the Community: Cross-Sectional Analysis \\ from ELSA-Brasil
}

\author{
Itamar S. Santos, ${ }^{10}$ Paulo A. Lotufo, ${ }^{1}$ Luisa Brant, ${ }^{2}{ }^{10}$ Marcelo M. Pinto-Filho, ${ }^{3}{ }^{(10}$ Alexandre da Costa Pereira, ${ }^{4}$ \\ Sandhi Maria Barreto, ${ }^{5}$ Antonio L. Ribeiro, ${ }^{6}{ }^{\circledR}$ G Neil Thomas, ${ }^{7}$ Gregory Y. H. Lip, ${ }^{8,9,10}{ }^{\text {Isabela M. Bensenor }}{ }^{1}$ \\ Hospital Universitário da Universidade de São Paulo, Centro de Pesquisa Clínica e Epidemiológica, ${ }^{1}$ São Paulo, SP - Brasil \\ Faculdade de Medicina da Universidade Federal de Minas Gerais, Departamento de Clínica Médica, ${ }^{2}$ Belo Horizonte, MG - Brasil \\ Faculdade de Medicina da Universidade Federal de Minas Gerais - Programa de Pós-Graduação em Infectologia e Medicina Tropical, ${ }^{3}$ Belo \\ Horizonte, MG - Brasil \\ Instituto do Coração do Hospital das Clínicas da Faculdade de Medicina da Universidade de São Paulo - Laboratório de Genética e \\ Cardiologia Molecular, ${ }^{4}$ São Paulo, SP - Brasil \\ Universidade Federal de Minas Gerais, ${ }^{5}$ Belo Horizonte, MG - Brasil \\ Universidade Federal de Minas Gerais - Centro de Telessaúde - Hospital das Clínicas - UFMG, ${ }^{6}$ Belo Horizonte, MG - Brasil \\ University of Birmingham College of Medical and Dental Sciences - Institute of Applied Health Research, ${ }^{7}$ Birmingham - Reino Unido da Grã-Bretanha \\ University of Liverpool - Liverpool Centre for Cardiovascular Science, ${ }^{8}$ Liverpool, Merseyside - Reino Unido da Grã-Bretanha \\ Liverpool Heart \& Chest Hospital, ${ }^{9}$ Liverpool - Reino Unido da Grã-Bretanha \\ Aalborg Universitet - Aalborg Thrombosis Research Unit, ${ }^{10}$ Aalborg - Dinamarca
}

\section{Resumo}

Fundamento: A fibrilação ou flutter atrial (FFA) é a arritmia cardíaca sustentada mais comum. Existem poucos dados sobre a epidemiologia da FFA na América do Sul.

Objetivo: O presente estudo procurou descrever a epidemiologia clínica da FFA e o uso de anticoagulantes na avaliação da linha de base do Estudo Longitudinal da Saúde do Adulto (ELSA-Brasil).

Métodos: Foram analisados dados de 13.260 participantes do ELSA-Brasil. A FFA foi definida pelo eletrocardiograma ou por autorrelato. Modelos de regressão logística foram construídos para analisar fatores associados à FFA. Este estudo também analisou se idade e sexo estavam associados ao uso de anticoagulantes para evitar acidente vascular cerebral. O nível de significância foi de $5 \%$.

Resultados: A idade mediana foi de 51 anos, e 7.213 (54,4\%) participantes eram mulheres. A FFA foi detectada em 333 (2,5\%) participantes. O aumento da idade (razão de chances [RC]:1,05; intervalo de confiança de 95\% [IC95\%]: 1,04-1,07), hipertensão (RC:1,44; IC95\%:1,14-1,81) coronariopatia (RC: 5,11; IC95\%:3,85-6,79), insuficiência cardíaca (RC:7,37; IC95\%:5,00-10,87) e febre reumática (RC:3,38; IC95\%:2,28-5,02) foram associadas à FFA. Dos 185 participantes com FFA e pontuação no $\mathrm{CHA}_{2} \mathrm{DS}_{2}-\mathrm{VASc} \geq 2$, apenas $20(\mathbf{1 0 , 8} \%)$ usavam anticoagulantes $(50,0 \%$ entre aqueles com FFA no eletrocardiograma de linha de base). $O$ uso de anticoagulantes nesse grupo foi associado a maior idade (1,8\% vs $17,7 \%$ naqueles com idade $\leq 54$ e $\geq$ 65 anos, respectivamente; $p=0,013)$. Observou-se uma tendência ao menor uso de anticoagulantes em mulheres $(7,1 \%$ vs. $16,4 \%$ em mulheres e homens, respectivamente; $p=0,055)$.

Conclusões: No recrutamento do ELSA-Brasil, 2,5\% dos participantes tinham FFA. $O$ baixo uso de anticoagulantes era comum, o que representa um desafio para os cuidados de saúde nesse cenário.

Palavras-chave: Fibrilação Atrial; Epidemiologia; Eletrocardiografia/métodos; Anticoagulantes; Estudo Longitudinal; Idoso; Acidente Vascular Cerebral; Embolia.

\footnotetext{
Abstract in South America.

Correspondência: Itamar S. Santos •

Universidade de São Paulo - Av. Prof. Lineu Prestes, 2565. CEP 05508-000, São Paulo, SP - Brasil

E-mail: itamarss@usp.br

Artigo recebido em 02/12/2019, revisado em 15/07/2020, aceito em 16/08/2020
}

Background: Atrial fibrillation or flutter (AFF) is the most common sustained cardiac arrhythmia. Limited data can be found on AFF epidemiology

DOI: https://doi.org/10.36660/abc.20190873 


\section{Artigo Original}

Objective: The present study sought to describe the clinical epidemiology of AFF and the use of stroke prevention medication in the Brazilian Longitudinal Study of Adult Health (ELSA-Brasil) baseline assessment.

Methods: This study analyzed data from 13,260 ELSA-Brasil participants. AFF was defined according to ECG recording or by self-report. Logistic regression models were built to analyze factors associated with AFF. This study also analyzed if age and sex were associated with anticoagulant use for stroke prevention. Significance level was set at $5 \%$.

Results: Median age was 51 years and 7,213 (54.4\%) participants were women. AFF was present in 333 (2.5\%) participants. Increasing age (odds ratio [OR]:1.05; 95\% confidence interval [95\% Cl]: 1.04-1.07), hypertension (OR:1.44; 95\%Cl: 1.14-1.81), coronary heart disease (OR: $5.11 ; 95 \% \mathrm{Cl}: 3.85-6.79)$, heart failure (OR:7.37; 95\%Cl: 5.00-10.87), and rheumatic fever (OR:3.38; 95\%Cl: 2.28-5.02) were associated with AFF. From 185 participants with AFF and a CHA2DS2-VASC score $\geq 2$, only 20 (10.8\%) used anticoagulants (50.0\% among those with AFF in the baseline ECG). Stroke prevention in this group was associated with a higher age (1.8\% vs $17.7 \%$ in those aged $\leq 54$ and $\geq 65$ years, respectively; $p=0.013)$. A trend towards a reduced anticoagulant use was observed in women (7.1\% vs. $16.4 \%$ in women and men, respectively; $p=0.055)$.

Conclusions: At the ELSA-Brasil baseline, 2.5\% of the participants had AFF. The lack of stroke prevention was common, which is an especially challenging point for healthcare in this setting.

Keywords: Atrial Fibrillation; Epidemiology; Electrocardiography/methods; Anticoagulants; Longitudinal Study; Aged; Stroke; Embolism.

Full texts in English - http://www.arquivosonline.com.br

\section{Introdução}

A fibrilação atrial é a arritmia cardíaca sustentada mais comum, com um risco vitalício aproximado de $25 \%^{1}$ e um total de 33,3 milhões de casos globalmente. ${ }^{2}$ Em São Paulo, Brasil, um estudo populacional ${ }^{3}$ identificou que 3,2\% dos homens e 2,0\% das mulheres com idade igual ou superior a 65 anos tinham fibrilação atrial. Outro grande banco de dados de pacientes em atenção primária com idade igual ou superior a 5 anos (71\% com idade $\geq 40$ anos), em Minas Gerais, no Brasil, ${ }^{4}$ identificou que 2,4\% dos homens e 1,3\% das mulheres tinham fibrilação atrial. Fatores de risco para fibrilação ou flutter atrial incluem idade, hipertensão, diabetes, tabagismo, obesidade, insuficiência cardíaca, doença valvar, e infarto do miocárdio. ${ }^{5-7}$

É recomendada a terapia de prevenção de tromboembolismo baseada em risco (incluindo acidente vascular cerebral) para pacientes com fibrilação ou flutter atrial (FFA). ${ }^{8,9}$ As pontuações $\mathrm{CHA}_{2} \mathrm{DS}_{2}-\mathrm{VASC}^{10}$ atualmente são a principal estratégia para avaliar o risco de tromboembolismo nesses indivíduos. ${ }^{11}$ A terapia antitrombótica oral é recomendada para pacientes com uma pontuação $\mathrm{CHA}_{2} \mathrm{DS}_{2}-\mathrm{VASc} \geq 2 .{ }^{9}$

A terapia anticoagulante oral, entretanto, ainda representa um desafio para a prática clínica, ${ }^{12,13}$ e uma proporção significativa da carga de morbidade da FFA ainda está associada ao acidente vascular cerebral incidente, ${ }^{14}$ especialmente em mulheres. ${ }^{15,16}$ Isso é ainda mais importante no Brasil, onde, embora os índices de incidentes de acidente vascular cerebral padronizada para a idade do país tenham caídos nos últimos anos, ${ }^{17}$ a mortalidade por acidente vascular cerebral é ainda alta, comparada a outros países sul-americanos, e tem um impacto maior nos estados menos desenvolvidos do país. ${ }^{17,18}$

O estudo Longitudinal de Saúde do Adulto (ELSA-Brasil) é uma grande coorte multicêntrica de indivíduos entre 35 e 74 anos de seis cidades brasileiras, com o objetivo de estudar a incidência e os fatores associados às doenças cardiovasculares e ao diabetes. O objetivo do presente estudo é descrever a frequência da FFA na linha de base do ELSA-Brasil e o uso de medicamentos de prevenção de acidente vascular cerebral nos participantes com FFA e com uma pontuação $\mathrm{CHA}_{2} \mathrm{DS}_{2}-\mathrm{VASc} \geq 2$.

\section{Métodos}

\section{Configuração e desenho do estudo}

O desenho do ELSA-Brasil ${ }^{19}$ e o perfil de coorte $^{20}$ estão detalhados em outro local. Resumidamente, o ELSA-Brasil é um estudo coorte de 15.105 funcionários públicos de seis cidades brasileiras (São Paulo, Belo Horizonte, Porto Alegre, Salvador, Rio de Janeiro, e Vitória). As avaliações de linha de base foram realizadas entre agosto de 2008 e dezembro de 2010 e incluíram entrevistas presenciais realizadas por pessoal treinado, utilizando exames clínicos, laboratoriais e de imagem. Desde a linha de base, todos os participantes receberam um contato telefone anual para acompanhamento. Quatro anos após o recrutamento (2012-2014), todos os participantes foram convidados a passar por uma reavaliação local, que incluiu questionários e avaliações clínicas e laboratoriais. Essa reavaliação teve a presença de 14.014 $(92,8 \%)$ participantes. O termo de consentimento informado foi obtido de todos os participantes. O protocolo do estudo está de acordo com a Resolução № 466/2012 do Conselho Nacional de Saúde e foi aprovado pelo Comitê de Análise Institucional de cada centro participante.

\section{Diagnóstico da fibrilação ou flutter atrial}

Os métodos de registro dos traçados de eletrocardiogramas (ECG) no ELSA-Brasil estão detalhados em outro artigo. ${ }^{21} \mathrm{O}$ eletrocardiograma na linha de base foi realizado em cada centro, utilizando-se um dispositivo Burdick Atria 6100, calibrado a $10 \mathrm{~mm} / \mathrm{mV}$ e uma velocidade de $25 \mathrm{~mm} /$ segundo. Os registros foram transmitidos ao centro de leitura no Centro de Investigação em Minas Gerais. As análises seguiram o sistema de Glasgow, ${ }^{22}$ e foram codificadas de acordo com o Minnesota Coding System. Códigos selecionados (incluindo FFA) foram analisados manualmente por uma equipe treinada. Além disso, durante a reavaliação local realizada em 2012-2014, a seguinte pergunta foi feita aos participantes: "Algum médico já disse que você tem/tinha fibrilação atrial?" Os participantes que responderam "sim" a essa pergunta também tiveram que responder "Quantos anos você tinha a primeira vez que um médico disse que você tem/tinha fibrilação atrial?" 
O presente estudo definiu o diagnóstico de FFA na linha de base se o participante (a) tivesse um registro de eletrocardiograma com FFA na avaliação de linha de base do ELSA-Brasil $(n=48$ ) ou (b) indicasse que tinha tido um diagnóstico de fibrilação atrial numa idade inferior à sua, no momento do recrutamento para o ELSA-Brasil $(n=285)$. A maioria das análises são apresentadas para todos os casos de FFA e de acordo com cada critério de definição.

\section{Amostra do estudo}

Este estudo incluiu participantes do ELSA-Brasil para os quais o diagnóstico de FFA na linha de base poderia ser avaliado. Dos 15.105 participantes do ELSA-Brasil, 663 $(4,4 \%)$ foram excluídos por não terem uma leitura válida de eletrocardiograma na linha de base, nem uma resposta válida sobre FFA durante a reavaliação local de 2012-2014, e $1.182(7,8 \%)$ foram excluídos porque tinham uma leitura válida de eletrocardiograma na linha de base sem FFA, mas não tinham uma resposta válida sobre FFA na reavaliação local de 2012-2014 (prejudicando a avaliação de FFA paroxística). Portanto, nossa amostra principal consistiu em 13.260 (87,8\%) participantes.

\section{Outras variáveis}

Idade, sexo, raça, grau de escolaridade, renda familiar mensal, e tabagismo foram autorrelatados e estratificados adequadamente. A renda mensal foi convertida em dólares americanos (utilizando uma taxa de câmbio de BRL 2,00 = USD 1,00 , com base na taxa de câmbio na linha de base). A raça foi definida, conforme utilizada no Censo Nacional Brasileiro, ou seja, preta, parda, branca, amarela ou indígena. As medições antropométricas no ELSA-Brasil foram avaliadas utilizando técnicas padronizadas, ${ }^{23}$ e o índice de massa corporal (IMC) foi calculado dividindo-se o pelo pela altura ao quadrado. $\mathrm{O}$ IMC foi categorizado como normal $\left(<25 \mathrm{~kg} / \mathrm{m}^{2}\right)$, sobrepeso $\left(\geq 25 \mathrm{~kg} / \mathrm{m}^{2}\right.$ e $\left.<30 \mathrm{~kg} / \mathrm{m}^{2}\right)$, e obesidade $\left(\geq 30 \mathrm{~kg} / \mathrm{m}^{2}\right)$. Hipertensão foi definida pelo relato de uso de medicamento anti-hipertensivos, pressão arterial sistólica $\geq 140 \mathrm{mmHg}$, ou pressão arterial diastólica $\geq 90 \mathrm{mmHg}$ no momento da avaliação de linha de base do ELSA-Brasil. Diabetes foi definido pelo histórico médico de diabetes mellitus, relato de uso de medicamentos para tratar diabetes mellitus, glicemia em jejum $\geq 126 \mathrm{mg} / \mathrm{dl}$, níveis de $\mathrm{HbA} 1 \mathrm{c} \geq 6,5 \%$, ou um teste oral de tolerância à glicose de 2 horas $\geq 200 \mathrm{mg} / \mathrm{dl}$. Dislipidemia foi definida pelo relato do uso de tratamento de redução lipídica ou um nível de colesterol LDL $\geq 130$ mg/dl. Obesidade abdominal foi definida como uma circunferência $>88 \mathrm{~cm}$ em mulheres e $102 \mathrm{~cm}$ em homens. Diagnósticos médicos anteriores de insuficiência cardíaca, acidente vascular cerebral, febre reumática, e evento tromboembólico foram avaliados a partir de autorrelato. Coronariopatia foi definida por um diagnóstico médico anterior de infarto do miocárdio ou angina, ou por revascularização coronária anterior. As aferições de pressão tornozelo-braquial para cálculo de índice tornozelo-braquial (ITB) são descritas detalhadamente por Miname et al. ${ }^{24}$. De acordo com os achados desse estudo, o ITB foi calculado como a proporção entre a pressão sistólica mínima do tornozelo e a pressão sistólica máxima no braço, para obter uma sensibilidade maior. Doença arterial periférica foi definida quando o ITB era $<1,0 .{ }^{24,25}$ Para dados sobre o uso de medicamentos, além dos questionários, solicitou-se que os pacientes trouxessem para a linha de base, todos os medicamentos, com receita médica ou não, que eles estivessem tomando no momento. ${ }^{26} \mathrm{O}$ uso de anticoagulantes foi definido como o uso de um medicamento com código Anatomical Therapeutic Chemical (ATC) B01AA ou B01AB. O uso de antiagregantes plaquetários foi definido como o uso de medicamentos com código ATC B01AC. Para participantes sem coronariopatia ou acidente vascular cerebral, o risco de doença cardiovascular aterosclerótica (DCVAS) de 10 anos foi calculado de acordo com as diretrizes de Avaliação de Risco Cardiovascular da ACC/AHA de 2013. ${ }^{27}$ As pontuações $\mathrm{CHA}_{2} \mathrm{DS}_{2}$-VASc foram calculadas de acordo com Lip et al. ${ }^{11}$ e categorizadas como 0 ou 1 pontos, 2 ou 3 pontos, e $\geq 4$ pontos.

\section{Análise estatística}

As variáveis categóricas são apresentadas como contagens e proporções, e são comparadas usando-se o teste Quiquadrado ou o teste exato de Fisher. As variáveis contínuas (idade, índice de massa corporal, índice tornozelo-braquial, e risco de DCVAS de 10 anos) têm distribuição não normal ( $p<0,001$ para todas, usando o teste Anderson-Darling), e são apresentadas como medianas e faixas interquartis, e são comparadas entre os grupos, usando o teste $U$ de MannWhitney. Modelos de regressão logística brutos padronizados para a idade e o sexo foram construídos para analisar se o diagnóstico de FFA na linha de base do ELSA-Brasil estava associado a idade, sexo, raça, grau de escolaridade, renda mensal, tabagismo, diagnósticos de hipertensão, diabetes e dislipidemia, índice de massa corporal, obesidade abdominal, doença arterial periférica, coronariopatia, insuficiência cardíaca, acidente vascular cerebral, febre reumática, e um risco de DCC de 10 anos $>10 \%$. A regressão logística binária foi usada para analisar a associação com todos os casos de FFA, enquanto a regressão logística multinomial foi usada para analisar a associação com casos de FFA de acordo com cada critério de definição (por registro de eletrocardiograma ou autorrelato) separadamente. O nível de significância foi definido em $5 \%$, e o software $R$, versão 3.5.1, foi usado em todas as análises.

\section{Resultados}

Entre os 13.260 participantes incluídos nas análises, 333 (2,5\%) tinham FFA na linha de base do ELSA-Brasil, 176/7.213 $(2,4 \%)$ eram mulheres e $157 / 6.047(2,6 \%)$ eram homens. De acordo com a faixa etária, a frequência de diagnóstico de FFA foi $1,2 \%, 2,2 \%, 2,9 \%$, e $5,4 \%$ para as idades $<45$, 45 $54,55-64$, e > 64 anos, respectivamente. A Tabela 1 mostra as características das amostras do estudo de acordo com a presença de diagnóstico de FFA na linha de base do ELSABrasil. Os indivíduos não brancos representaram 47,9\% da amostra, e a maioria dos participantes tinham ensino superior completo ou mais.

A Tabela 2 mostra razões de chance padronizadas para idade e sexo para a associação com a presença de diagnóstico de FFA na linha de base do ELSA-Brasil. Considerando todos 
Tabela 1 - Características das amostras do estudo de acordo com a presença de diagnóstico de FFA na linha de base do ELSA-Brasil

\begin{tabular}{|c|c|c|c|c|c|c|}
\hline & \multirow[b]{2}{*}{$\begin{array}{l}\text { Sem fibrilação ou } \\
\text { flutter atrial. } \\
(\mathrm{N}=12.927)\end{array}$} & \multicolumn{3}{|c|}{ Fibrilação ou flutter atrial } & \multirow[b]{2}{*}{$\begin{array}{c}\text { Total } \\
(\mathrm{N}=13.260)\end{array}$} & \multirow[b]{2}{*}{ p-valor } \\
\hline & & $\begin{array}{l}\text { Por registro de } \\
\text { eletrocardiograma } \\
(\mathrm{N}=48)\end{array}$ & $\begin{array}{l}\text { Apenas por } \\
\text { autorrelato } \\
(\mathrm{N}=285)\end{array}$ & $\begin{array}{c}\text { Todos os casos } \\
\text { de FFA } \\
(\mathrm{N}=333)\end{array}$ & & \\
\hline Idade (anos; mediana [P25 - P75]) & $51,0[45,0-58,0]$ & $61,5[56,0-71,0]$ & $54,0[49,0-62,0]$ & $56,0[49,0-63,0]$ & $51,0[45,0-58,0]$ & $<0,001 \ddagger$ \\
\hline Sexo feminino (N (\%)) & $7.037(54,4 \%)$ & $18(37,5 \%)$ & $158(55,4 \%)$ & $176(52,9 \%)$ & $7.213(54,4 \%)$ & $0,61 \dagger$ \\
\hline \multicolumn{7}{|l|}{ Raça (N (\%)) } \\
\hline Branco & $6.652(52,1 \%)$ & $29(61,7 \%)$ & $154(54,6 \%)$ & $183(55,6 \%)$ & $6.835(52,1 \%)$ & \multirow{4}{*}{$0,039 \dagger$} \\
\hline Pardo & $3.608(28,2 \%)$ & $11(23,4 \%)$ & $74(26,2 \%)$ & $85(25,8 \%)$ & $3.693(28,2 \%)$ & \\
\hline Negro & $2.069(16,2 \%)$ & $7(14,9 \%)$ & $35(12,4 \%)$ & $42(12,8 \%)$ & $2.111(16,1 \%)$ & \\
\hline Outros & $449(3,5 \%)$ & $0(0,0 \%)$ & $19(6,7 \%)$ & $19(5,8 \%)$ & $468(3,6 \%)$ & \\
\hline \multicolumn{7}{|l|}{ Grau de escolaridade (N (\%)) } \\
\hline Ensino médio incompleto & $1.529(11,8 \%)$ & $13(27,1 \%)$ & $34(11,9 \%)$ & $47(14,1 \%)$ & $1.576(11,9 \%)$ & \multirow{3}{*}{$0,10 \dagger$} \\
\hline Ensino médio completo & $4.443(34,4 \%)$ & $12(25,0 \%)$ & $85(29,8 \%)$ & $97(29,1 \%)$ & $4.540(34,2 \%)$ & \\
\hline Ensino superior ou mais & $6.955(53,8 \%)$ & $23(47,9 \%)$ & $166(58,2 \%)$ & $189(56,8 \%)$ & $7.144(53,9 \%)$ & \\
\hline \multicolumn{7}{|l|}{ Renda mensal (N (\%)) } \\
\hline$<$ USD\$ 1245 & $3.357(26,1 \%)$ & $15(31,2 \%)$ & $58(20,4 \%)$ & $73(22,0 \%)$ & $3.430(26,0 \%)$ & \multirow{3}{*}{$0,001 \dagger$} \\
\hline USD\$ 1245-3319 & $5.679(44,1 \%)$ & $12(25,0 \%)$ & $115(40,5 \%)$ & $127(38,3 \%)$ & $5.806(43,9 \%)$ & \\
\hline$\geq$ USD $\$ 3320$ & $3.845(29,9 \%)$ & $21(43,8 \%)$ & $111(39,1 \%)$ & $132(39,8 \%)$ & $3.977(30,1 \%)$ & \\
\hline \multicolumn{7}{|l|}{ Tabagismo (N (\%)) } \\
\hline Nunca fumou & $7.467(57,8 \%)$ & $29(60,4 \%)$ & $161(56,5 \%)$ & $190(57,1 \%)$ & $7.657(57,7 \%)$ & \multirow{3}{*}{$0,75 \dagger$} \\
\hline Ex-fumante & $3.822(29,6 \%)$ & $13(27,1 \%)$ & $91(31,9 \%)$ & $104(31,2 \%)$ & $3.926(29,6 \%)$ & \\
\hline Fumante & $1.637(12,7 \%)$ & $6(12,5 \%)$ & $33(11,6 \%)$ & $39(11,7 \%)$ & $1.676(12,6 \%)$ & \\
\hline Hipertensão (N (\%)) & $4.463(34,5 \%)$ & $30(62,5 \%)$ & $136(47,7 \%)$ & $166(49,8 \%)$ & $4.629(34,9 \%)$ & $<0,001 \dagger$ \\
\hline Diabetes (N (\%)) & $2.438(18,9 \%)$ & $15(31,2 \%)$ & $66(23,2 \%)$ & $81(24,3 \%)$ & $2.519(19,0 \%)$ & $0,015 \dagger$ \\
\hline Dislipidemia (N (\%)) & $7.489(58,0 \%)$ & $27(56,2 \%)$ & $188(66,0 \%)$ & $215(64,6 \%)$ & $7.704(58,1 \%)$ & $0,019 \dagger$ \\
\hline $\begin{array}{l}\text { Índice de massa corporal } \\
\left(\mathrm{kg} / \mathrm{m}^{2} ; \text { mediana [P25 - P75]) }\right.\end{array}$ & $26,3[23,7-29,5]$ & $27,4[25,4-30,4]$ & $26,2[23,9-30,2]$ & $26,5[24,1-30,3]$ & $26,3[23,7-29,6]$ & $0,35 \ddagger$ \\
\hline \multicolumn{7}{|l|}{$\begin{array}{l}\text { Classificação do índice de massa } \\
\text { corporal (N (\%)) }\end{array}$} \\
\hline Normal & $4.791(37,1 \%)$ & $10(20,8 \%)$ & $104(36,5 \%)$ & $114(34,2 \%)$ & $4.905(37,0 \%)$ & \multirow{3}{*}{$0,057 \dagger$} \\
\hline Sobrepeso & $5.238(40,5 \%)$ & $24(50,0 \%)$ & $102(35,8 \%)$ & $126(37,8 \%)$ & $5.364(40,5 \%)$ & \\
\hline Obesidade & $2.892(22,4 \%)$ & $14(29,2 \%)$ & $79(27,7 \%)$ & $93(27,9 \%)$ & $2.985(22,5 \%)$ & \\
\hline $\begin{array}{l}\text { Obesidade abdominal } \\
(\mathrm{N}(\%))\end{array}$ & $4.656(36,0 \%)$ & $20(41,7 \%)$ & $113(39,6 \%)$ & $133(39,9 \%)$ & $4.789(36,1 \%)$ & $0,16 \dagger$ \\
\hline $\begin{array}{l}\text { Índice tornozelo-braquial (mediana } \\
\text { [P25 - P75]) }\end{array}$ & $1,18[1,12-1,23]$ & $1,13[1,04-1,20]$ & $1,17[1,11-1,24]$ & $1,16[1,10-1,23]$ & $1,18[1,12-1,23]$ & $0,008 \ddagger$ \\
\hline Doença arterial periférica (N (\%)) & $403(3,1 \%)$ & $6(12,5 \%)$ & $12(4,2 \%)$ & $18(5,4 \%)$ & $421(3,2 \%)$ & $0,025 \dagger$ \\
\hline Uso de anticoagulantes (N (\%)) & $37(0,3 \%)$ & $19(39,6 \%)$ & $5(1,8 \%)$ & $24(7,2 \%)$ & $61(0,5 \%)$ & $<0,001 \dagger$ \\
\hline $\begin{array}{l}\text { Uso de antiagregantes } \\
\text { plaquetários (N (\%)) }\end{array}$ & $706(5,5 \%)$ & $7(14,6 \%)$ & $45(15,8 \%)$ & $52(15,6 \%)$ & $758(5,7 \%)$ & $<0,001 \dagger$ \\
\hline $\begin{array}{l}\text { Uso de anticoagulantes e/ou } \\
\text { antiagregantes plaquetários (N (\%)) }\end{array}$ & $741(5,7 \%)$ & $26(54,2 \%)$ & $50(17,5 \%)$ & $76(22,8 \%)$ & $817(6,2 \%)$ & $<0,001 \dagger$ \\
\hline Coronariopatia (N (\%)) & $536(4,2 \%)$ & $7(14,6 \%)$ & $67(23,5 \%)$ & $74(22,2 \%)$ & $610(4,6 \%)$ & $<0,001 \dagger$ \\
\hline Insuficiência cardíaca (N (\%)) & $160(1,2 \%)$ & $10(20,8 \%)$ & $26(9,2 \%)$ & $36(10,8 \%)$ & $196(1,5 \%)$ & $<0,001 \dagger$ \\
\hline Acidente vascular cerebral (N (\%)) & $153(1,2 \%)$ & $1(2,1 \%)$ & $7(2,5 \%)$ & $8(2,4 \%)$ & $161(1,2 \%)$ & $0,067 \dagger$ \\
\hline Febre reumática (N (\%)) & $352(2,7 \%)$ & $7(14,6 \%)$ & $23(8,1 \%)$ & $30(9,0 \%)$ & $382(2,9 \%)$ & $<0,001 \dagger$ \\
\hline Evento tromboembólico (N (\%)) & $198(1,5 \%)$ & $4(8,3 \%)$ & $5(1,8 \%)$ & $9(2,7 \%)$ & $207(1,6 \%)$ & $0,11 \dagger$ \\
\hline
\end{tabular}




\section{Continuação}

\begin{tabular}{|c|c|c|c|c|c|c|}
\hline $\begin{array}{l}\text { Risco de DCVAS de } 10 \text { anos } \\
\text { (mediana [P25 - P75]) }\end{array}$ & $2,8 \%[1,1 \%-7,1 \%]$ & $\begin{array}{c}11,8 \%[4,2 \%- \\
18,5 \%]\end{array}$ & $2,7 \%[1,4 \%-7,9 \%]$ & $3,4 \%[1,5 \%$ - 10,4\%] & $2,8 \%[1,1 \%-7,2 \%]$ & $0,001 \ddagger$ \\
\hline $\begin{array}{l}\text { Risco de DCVAS de } 10 \text { anos > } \\
10 \%(\mathrm{~N}(\%))\end{array}$ & $2075(16,9 \%)$ & $23(57,5 \%)$ & $41(19,2 \%)$ & $64(25,2 \%)$ & $2139(17,1 \%)$ & $0,001 \dagger$ \\
\hline
\end{tabular}

Tabela 2 - Razões de chance padronizadas para idade e sexo (intervalos de confiança de 95\%) para a associação com fibrilação ou flutter atrial na linha de base do ELSA-Brasil

\begin{tabular}{|c|c|c|c|}
\hline \multirow[b]{2}{*}{ Variável } & \multicolumn{3}{|c|}{ Fibrilação ou flutter atrial } \\
\hline & $\begin{array}{l}\text { Por registro de } \\
\text { eletrocardiograma } \\
\qquad(\mathrm{N}=48)\end{array}$ & $\begin{array}{l}\text { Apenas por autorrelato } \\
\qquad(\mathrm{N}=285)\end{array}$ & $\begin{array}{c}\text { Todos os casos de FFA } \\
(\mathrm{N}=333)\end{array}$ \\
\hline Idade (incrementos de um ano) & $1,12[1,09-1,16]$ & $1,04(1,03-1,06) \dagger$ & $1,05(1,04-1,07) \dagger$ \\
\hline Sexo feminino & $0,53(0,30-0,96) \dagger$ & $1,05(0,83-1,33)$ & $0,95(0,76-1,18)$ \\
\hline \multicolumn{4}{|l|}{ Raça* } \\
\hline Branco & 1,0 (Referência) & 1,0 (Referência) & 1,0 (Referência) \\
\hline Pardo & $0,92(0,45-1,85)$ & $0,95(0,72-1,26)$ & $0,94(0,72-1,22)$ \\
\hline Negro & $(0,43-2,27)$ & $0,76(0,52-1,10)$ & $0,79(0,56-1,10)$ \\
\hline \multicolumn{4}{|l|}{ Grau de escolaridade } \\
\hline Ensino médio incompleto & $1,73(0,87-3,43)$ & $0,79(0,54-1,15)$ & $0,92(0,66-1,27)$ \\
\hline Ensino médio completo & $1,12(0,55-2,28)$ & $0,86(0,66-1,12)$ & $0,88(0,69-1,13)$ \\
\hline Ensino superior ou mais & 1,0 (Referência) & 1,0 (Referência) & 1,0 (Referência) \\
\hline \multicolumn{4}{|l|}{ Renda mensal } \\
\hline$<$ USD $\$ 1245$ & $1,27(0,64-2,51)$ & $0,67(0,48-0,93) \dagger$ & $0,74(0,55-0,99) \dagger$ \\
\hline USD\$ $1245-3319$ & $0,61(0,29-1,25)$ & $0,80(0,61-1,04)$ & $0,77(0,60-0,99) \dagger$ \\
\hline$\geq$ USD\$ $3320(\mathrm{~N}(\%))$ & 1,0 (Referência) & 1,0 (Referência) & 1,0 (Referência) \\
\hline \multicolumn{4}{|l|}{ Tabagismo } \\
\hline Nunca fumou & (Referência) & (Referência) & 1,0 (Referência) \\
\hline Ex-fumante & $0,57(0,29-1,12)$ & $0,98(0,75-1,27)$ & $0,90(0,70-1,16)$ \\
\hline Fumante & $1,00(0,42-2,40)$ & $0,93(0,64-1,36)$ & $0,93(0,65-1,32)$ \\
\hline Hipertensão & $1,65(0,89-3,03)$ & $1,41(1,10-1,81) \dagger$ & $1,44(1,14-1,81) \dagger$ \\
\hline Diabetes & $1,11(0,60-2,08)$ & $1,06(0,80-1,41)$ & $1,07(0,82-1,39)$ \\
\hline Dislipidemia & $0,66(0,37-1,18)$ & $1,22(0,95-1,57)$ & $1,10(0,87-1,39)$ \\
\hline \multicolumn{4}{|c|}{ Classificação do índice de massa corporal } \\
\hline Normal & 1,0 (Referência) & 1,0 (Referência) & 1,0 (Referência) \\
\hline Sobrepeso & $1,88(0,90-3,95)$ & $0,85(0,64-1,12)$ & $0,94(0,72-1,21)$ \\
\hline Obesidade & $2,21(0,97-5,00)$ & $1,19(0,88-1,60)$ & $1,27(0,96-1,68)$ \\
\hline Obesidade abdominal & $1,15(0,63-2,08)$ & $1,04(0,81-1,33)$ & $1,05(0,83-1,32)$ \\
\hline Doença arterial periférica & $2,72(1,13-6,54) \dagger$ & $1,16(0,64-2,10)$ & $1,44(0,88-2,35)$ \\
\hline Doença arterial coronariana & $1,87(0,81-4,28)$ & $5,99(4,44-8,09) \dagger$ & $5,11(3,85-6,79) \dagger$ \\
\hline Insuficiência cardíaca & $11,67(5,58-24,40) \dagger$ & $6,51(4,19-10,11) \dagger$ & $7,37(5,00-10,87) \dagger$ \\
\hline Acidente vascular & $0,90(0,12-6,67)$ & $1,65(0,76-3,57)$ & $1,51(0,73-3,13)$ \\
\hline Febre reumática & $5,75(2,55-12,98) \dagger$ & $3,02(1,94-4,70) \dagger$ & $3,38(2,28-5,02) \dagger$ \\
\hline Risco de DCVAS de 10 anos $>10 \%$ & $1,56(0,63-3,85)$ & $0,78(0,50-1,21)$ & $0,95(0,65-1,40)$ \\
\hline
\end{tabular}

*A proporção pequena de indivíduos de outras raças na amostra impossibilitou a estimativa. FFA: Fibrilação ou flutter atrial. DCVAS: Doença cardiovascular aterosclerótica. As razões de chance e os p-valores foram obtidos a partir de modelos de regressão logística padronizados para idade e sexo. $t p<0,05$ 
os casos de FFA, idade mais alta $(p<0,001)$, renda mais alta $(p=0,044)$, e diagnósticos de hipertensão $(p=0,002)$, coronariopatia $(p<0,001)$, insuficiência cardíaca $(p<0,001)$, e febre reumática $(p<0,001)$ foram positivamente associados a FFA nos modelos padronizados. Restringindo os casos aos 48 participantes que apresentaram FFA no eletrocardiograma de linha de base, idade mais alta $(p<0,001)$, sexo masculino $(p=0,037)$, e diagnósticos de doença arterial periférica $(p=0,026)$, insuficiência cardíaca $(p<0,001)$, e febre reumática $(p<0,001)$ foram positivamente associados a FFA nos modelos padronizados. A Tabela suplementar 1 mostra os resultados de modelos brutos para essas associações.

Foi possível calcular as pontuações $\mathrm{CHA}_{2} \mathrm{DS}_{2}$-VASc para 331 (99,4\%) dos 333 participantes com diagnóstico de FFA na linha de base (Tabela 3). Como esperado, os indivíduos com pontuações $\mathrm{CHA}_{2} \mathrm{DS}_{2}$-VASc mais altas eram mais velhos $(p<0,001)$, tinham uma probabilidade mais alta de serem mulheres $(p=0,002)$, e apresentaram um risco cardiovascular mais alto $(p<0,001)$. Entre os 185 participantes com FFA e pontuação no $\mathrm{CHA}_{2} \mathrm{DS}_{2}$-VASc $\geq 2$, apenas 20 (10,8\%) faziam tratamento com anticoagulantes $(16 / 32 ; 50,0 \%$ quando os casos eram restritos aos 48 participantes que apresentaram FFA no eletrocardiograma de linha de base).

A Tabela 4 mostra a prevalência do uso de anticoagulantes orais em participantes com FFA e uma pontuação $\mathrm{CHA}_{2} \mathrm{DS}_{2}-$ VASc $\geq 2$, de acordo com idade e sexo. Considerando todos os casos de FFA, o uso de anticoagulantes foi mais frequente nos participantes com idade mais alta $(p=0,013)$, e uma tendência para uso mais baixo de anticoagulantes em mulheres $(p=0,055)$. A Tabela suplementar 2 mostra que a frequência do uso de anticoagulantes em homens era numericamente mais alta do que em mulheres para todas as faixas etárias. Entretanto, não foram descobertas diferenças significativas nem ao analisar todos os casos de FFA nem ao restringi-los aos participantes que apresentaram FFA no eletrocardiograma da linha de base.

\section{Discussão}

\section{Contextualização e discussão dos principais achados}

Em nossa amostra de coorte, 2,5\% dos participantes incluídos apresentaram FFA na linha de base. Idade, sexo masculino, renda, e diagnósticos de hipertensão, coronariopatia, doença arterial periférica, insuficiência cardíaca e febre reumática foram associados positivamente ao FFA. Segundo, a maioria dos participantes com FFA e uma pontuação $\mathrm{CHA}_{2} \mathrm{DS}_{2}-\mathrm{VASC} \geq 2$ não foi tratada com anticoagulantes e/ou antiagregantes plaquetários. Em terceiro lugar, o uso de anticoagulante oral foi mais comum naqueles com idade mais alta, enquanto as mulheres eram tratadas menos frequentemente.

A comparação da prevalência de FFA em amostras diferentes é desafiadora devido às heterogeneidades no desenho do estudo, no cenário do estudo, e na distribuição de idade e sexo no recrutamento. Uma análise da epidemiologia global da fibrilação atrial ${ }^{28}$ detectou uma prevalência mundial relatada variando entre $0,1 \%$ e $6,0 \%$, dependendo da faixa etária e do sexo analisados. Embora este estudo relate uma frequência de diagnóstico de FFA semelhante na amostra,

Tabela 3 - Características dos participantes com fibrilação ou flutter atrial na linha de base do ELSA-Brasil de acordo com as pontuações CHA2DS2-VASc

\begin{tabular}{|c|c|c|c|c|c|c|c|}
\hline & \multicolumn{7}{|c|}{ Fibrilação ou flutter atrial } \\
\hline & \multicolumn{2}{|c|}{ Por registro de eletrocardiograma } & \multicolumn{2}{|c|}{ Apenas por autorrelato } & \multicolumn{3}{|c|}{ Todos os casos de FFA } \\
\hline & $\begin{array}{c}\text { Pontuação } \\
\text { CHA2DS2-VASc } \\
<2 \\
(\mathrm{~N}=16)\end{array}$ & $\begin{array}{c}\text { Pontuação } \\
\text { CHA2DS2-VASc } \\
\geq 2 \\
(\mathrm{~N}=32)\end{array}$ & $\begin{array}{c}\text { Pontuação } \\
\text { CHA2DS2-VASc } \\
<2 \\
(\mathrm{~N}=130)\end{array}$ & $\begin{array}{c}\text { Pontuação } \\
\text { CHA2DS2-VASc } \\
\geq 2 \\
(\mathrm{~N}=153)\end{array}$ & $\begin{array}{c}\text { Pontuação } \\
\text { CHA2DS2-VASc } \\
<2 \\
(\mathrm{~N}=146)\end{array}$ & $\begin{array}{c}\text { Pontuação } \\
\text { CHA2DS2-VASc } \\
\geq 2 \\
(\mathrm{~N}=185)\end{array}$ & p-valor \\
\hline $\begin{array}{l}\text { Idade (anos; mediana } \\
\text { [P25 - P75]) }\end{array}$ & $56,0[49,5-61,2]$ & $67,5[58,0-71,2]$ & $49,5[45,0-56,0]$ & $59,0[53,0-66,0]$ & $50,0[45,0-57,0]$ & $60,0[53,0-68,0]$ & $<0,001 \ddagger$ \\
\hline Sexo feminino (N (\%)) & $3(18,8 \%)$ & $15(46,9 \%)$ & $60(46,2 \%)$ & $97(63,4 \%)$ & $63(43,2 \%)$ & $112(60,5 \%)$ & $0,002 \dagger$ \\
\hline Coronariopatia (N (\%)) & $0(0,0 \%)$ & $7(21,9 \%)$ & $2(1,5 \%)$ & $64(41,8 \%)$ & $2(1,4 \%)$ & $71(38,4 \%)$ & $<0,001 \dagger$ \\
\hline $\begin{array}{l}\text { Acidente vascular } \\
\text { cerebral }(\mathrm{N}(\%))\end{array}$ & $0(0,0 \%)$ & $1(3,1 \%)$ & $0(0,0 \%)$ & $7(4,6 \%)$ & $0(0,0 \%)$ & $8(4,3 \%)$ & $0,010 ¥$ \\
\hline $\begin{array}{l}\text { Risco de DCVAS em } \\
10 \text { anos }>10 \%(\mathrm{~N}(\%))\end{array}$ & $7(43,8 \%)$ & $16(66,7 \%)$ & $15(11,7 \%)$ & $26(30,6 \%)$ & $22(15,3 \%)$ & $42(38,5 \%)$ & $<0,001 \dagger$ \\
\hline $\begin{array}{l}\text { DCVAS anterior ou } \\
\text { Risco de DCVAS em } \\
10 \text { anos anterior }>10 \%\end{array}$ & $7(43,8 \%)$ & $24(75,0 \%)$ & $17(13,1 \%)$ & $94(61,4 \%)$ & $24(16,4 \%)$ & $118(63,8 \%)$ & $<0,001 \dagger$ \\
\hline $\begin{array}{l}\text { Uso de anticoagulantes } \\
(\mathrm{N}(\%))\end{array}$ & $3(18,8 \%)$ & $16(50,0 \%)$ & $1(0,8 \%)$ & $4(2,6 \%)$ & $4(2,7 \%)$ & $20(10,8 \%)$ & $0,005 ¥$ \\
\hline $\begin{array}{l}\text { Uso de antiagregantes } \\
\text { plaquetários (N (\%)) }\end{array}$ & $2(12,5 \%)$ & $5(15,6 \%)$ & $2(1,5 \%)$ & $43(28,1 \%)$ & $4(2,7 \%)$ & $48(25,9 \%)$ & $<0,001 \dagger$ \\
\hline
\end{tabular}

O risco de DCVAS de 10 anos é definido apenas para participantes sem histórico de coronariopatia ou acidente vascular cerebral. FFA: Fibrilação ou flutter atrial. DCVAS: Doença cardiovascular aterosclerótica. Os p-valores são apresentados para comparação entre os grupos com pontuação $\mathrm{CHA}_{2} D S_{2}-V A S C<2(N=146)$ e com pontuação $C H A_{2} D S_{2}-V A S C \geq 2$ ( $N=185$ ) entre todos os casos de FFA, utilizando os testes $\uparrow$ Qui-quadrado, $¥ U$ de Mann-Whitney $U$ ou $¥$ exato de Fisher. 
Tabela 4 - Frequência do uso de anticoagulantes, de acordo com idade e sexo, em participantes com fibrilação ou flutter atrial e uma pontuação $\mathrm{CHA}_{2} \mathrm{DS}_{2}-\mathrm{VASc} \geq 2$, na linha de base do ELSA-Brasil

\begin{tabular}{|c|c|c|c|c|}
\hline \multirow[b]{2}{*}{ Variável } & \multicolumn{4}{|c|}{ Fibrilação ou flutter atrial e uma pontuação $\mathrm{CHA}_{2} \mathrm{DS}_{2}-\mathrm{VASc} \geq 2$} \\
\hline & $\begin{array}{l}\text { Por registro de } \\
\text { eletrocardiograma } \\
(\mathrm{N}=32)\end{array}$ & $\begin{array}{l}\text { Apenas por autorrelato } \\
\qquad(\mathrm{N}=153)\end{array}$ & $\begin{array}{c}\text { Todos os casos de FFA } \\
(\mathrm{N}=185)\end{array}$ & p-valor \\
\hline \multicolumn{4}{|l|}{ Idade (anos) } & \multirow{3}{*}{0,013} \\
\hline$\leq 54$ & $1 / 3(33,3 \%)$ & $0 / 52(0,0 \%)$ & $1 / 55(1,8 \%)$ & \\
\hline$\geq 65$ & $9 / 18(50,0 \%)$ & $2 / 44(4,5 \%)$ & $11 / 62(17,7 \%)$ & \\
\hline \multicolumn{4}{|l|}{ Sexo } & \multirow{3}{*}{0,055} \\
\hline Masculino & $9 / 17(52,9 \%)$ & $3 / 56(5,4 \%)$ & 12 / $73(16,4 \%)$ & \\
\hline Feminino & $7 / 15(46,7 \%)$ & $1 / 97(1,0 \%)$ & $8 / 112(7,1 \%)$ & \\
\hline
\end{tabular}

quando comparado com os estudos brasileiros citados, ${ }^{3,4}$ esses estudos têm diferenças marcadas e são complementares. Por exemplo, Kawabata-Yoshihara et al. ${ }^{3}$ realizaram um estudo de recrutamento porta a porta sistemático de indivíduos com idade igual ou superior a 65 anos, enquanto Marcolino et al. ${ }^{4}$ usou um grande banco de dados de eletrocardiogramas da Rede de Teleassistência de Minas Gerais, composto de indivíduos com idade igual ou superior a 5 anos que receberam cuidados médicos em unidades de atenção primária de saúde. Ambos os estudos consideraram o diagnóstico de FFA de acordo com os registros de eletrocardiograma na avaliação. Ao comparar os achados desses estudos com os achados do presente estudo, detectou-se uma frequência mais alta de FFA quando faixas etárias semelhantes foram comparadas. Provavelmente isso foi influenciado pelas definições de caso, já que o presente estudo também incluiu um diagnóstico médico autorrelatado como critério diagnóstico. Entretanto, considerar apenas os traçados de eletrocardiograma durante a avaliação e excluir o histórico médico das definições pode levar a uma especificidade mais alta e reduzir erros de informação autorrelatada, mas também pode subestimar os índices de prevalência devido ao subreconhecimento da fibrilação atrial paroxística.

A associação entre insuficiência cardíaca e fibrilação atrial tem importância clínica. Healey et al. ${ }^{12}$ analisaram dados de 15.400 indivíduos com FFA em 47 países. Depois de um ano de acompanhamento, ocorreram 11\% de mortes no coorte, e a insuficiência cardíaca foi a causa de morte mais comum (30\%). Da mesma maneira, os indivíduos com doença arterial periférica e FFA têm um risco mais alto de acidente vascular cerebral, comparados com aqueles que só têm FFA. ${ }^{29}$ É importante observar que, em décadas recentes, a doença cardíaca reumática tem diminuído como causa de mortalidade no Brasil, ${ }^{30}$ e, embora novos casos no país sejam menos comuns, pessoas atualmente de meia idade e adultos mais velhos podem ter tido febre reumática durante a infância. Portanto, a prevalência e a mortalidade devidas à doença cardíaca reumática no Brasil não podem ser desprezadas, ${ }^{31}$ o que é reforçado por nosso achado de que $9,0 \%$ dos indivíduos com FFA nessa amostra de estudo (e 2,9\% em geral) apresentaram um histórico médico de febre reumática.

Um achado notável do presente estudo foi que 89,2\% de todos os participantes com FFA e uma pontuação $\mathrm{CHA}_{2} \mathrm{DS}_{2}$ $\mathrm{VASc} \geq 2$ não foram tratados com anticoagulante. Isso é mais importante, considerando que os participantes do ELSA-Brasil tinham um grau de escolaridade médio e renda mais altos, quando comparados à população brasileira geral. ${ }^{20}$ Embora os diagnósticos autorrelatados possam ter influenciado nossos achados de baixos índices de prevenção de acidente vascular cerebral, quando limitamos as análises a participantes com FFA documentado no registro de eletrocardiograma de linha de base, metade deles ainda não tinha recebido anticoagulante.

Embora preocupantes, esses baixos índices de prescrição para prevenção de acidente vascular cerebral não são exclusividade de nossa amostra. Um estudo semelhante realizado por Healey et al. ${ }^{12}$ descreveu que, por região geográfica, de 32\% (América do Norte, Europa Ocidental e Oriente Médio) a 70\% (China) pacientes com indicação por diretriz não receberam tratamento com anticoagulante oral. Na América do Sul, onde o ELSA-Brasil está localizado, a frequência da não prescrição era $55 \%$. Ogilvie et al. ${ }^{32}$ relataram uma análise sistemática de estudos relatando o uso de anticoagulantes orais em casos de fibrilação atrial fora de ensaios clínicos. Eles identificaram que 21/29 (72,4\%) estudos em pacientes com acidente vascular cerebral ou ataque isquêmico transitório prévio, bem como 5/9 (55,6\%) estudos em pacientes com alto risco tromboembólico, de acordo com escores de risco, relataram níveis de prescrição inferiores a $60 \%$.

A subprescrição de medicamentos para a prevenção de acidente vascular cerebral parece ser um problema mais grave em mulheres, ${ }^{33}$ o que está de acordo com nossos dados. Khurshid et al..$^{34}$ analisaram dados médicos eletrônicos de 4.388 pacientes com fibrilação atrial e detectaram que, comparadas aos homens, as mulheres receberam menos tratamento com anticoagulantes 1, 3 e 6 meses após o diagnóstico de fibrilação atrial. Além disso, Emdin et al. ${ }^{35}$ 
realizaram uma análise sistemática e metanálise de 30 estudos coorte e identificaram que, comparadas aos homens, mulheres com fibrilação atrial tinham um risco mais alto de mortalidade global, mortalidade cardiovascular, e acidente vascular cerebral. Esses autores destacam que, além da subutilização de medicamentos para prevenção de acidente vascular cerebral, outras explicações para resultados piores, tais como os índices mais altos de efeitos adversos dos tratamentos com anticoagulantes ${ }^{36}$ e antiarrítmicos ${ }^{37}$ em mulheres são igualmente plausíveis. Além disso, alguns autores destacaram um controle anticoagulante pior em mulheres que utilizam a varfarina. Por exemplo, Sullivan et al. ${ }^{38}$ analisaram dados de 4.060 participantes do estudo Atrial Fibrillation Follow-up Investigation of Rhythm Management (AFFIRM - Investigação de monitoramento da fibrilação atrial para controle do ritmo cardíaco), e concluíram que, comparadas aos homens, as mulheres em tratamento com varfarina passaram consideravelmente mais tempo utilizando doses inferiores à faixa terapêutica da razão normalizada internacional (RNI) (29\% vs 26\%). Também é importante notar que, a avaliação da linha de base do ELSABrasil ocorreu de 2008 a 2010. Nesse momento, o uso de anticoagulantes orais de ação direta para a prevenção de acidente vascular cerebral nos casos de FFA era muito raro.

\section{Pontos fortes e limitações}

Este estudo realmente tem alguns pontos fortes. Analisamos dados de uma amostra multicêntrica grande de indivíduos que não foram recrutados de cenários clínicos. Isso reduz possíveis vieses no estudo de fatores associados à fibrilação atrial e diagnósticos, e a prescrição de medicamentos para a prevenção de acidente vascular cerebral, aproximando nossa validade externa à população geral. O protocolo abrangente do ELSA-Brasil permitiu a análise do uso de medicamento de acordo com as pontuações de risco tromboembólico $\mathrm{CHA}_{2} \mathrm{DS}_{2}$-VASc. O estudo também deve ser interpretado dentro de seu contexto. Conforme afirmado anteriormente, esta definição do estudo de caso pode ter uma tendência a classificações erradas por informações autorrelatadas. Embora essa estratégia minimize o sub-reconhecimento da fibrilação atrial paroxística, é importante admitir que casos autorrelatados representaram uma proporção importante de todos os casos de FFA, e isso pode ter influenciado alguns de nossos resultados. Os dados sobre a frequência de indivíduos dentro da faixa terapêutica de RNI ou escores de risco de hemorragia não estavam disponíveis. Entretanto, acreditamos que o risco de hemorragia não é suficiente para explicar uma parte significativa da falta de prevenção de acidente vascular cerebral nessa amostra.

\section{Referências}

1. Lloyd-Jones DM, Wang TJ, Leip EP, Larson MG, Levy D, Vasan RS, et al. Lifetime risk for development of atrial fibrillation: the Framingham Heart Study. Circulation. 2004;110(9):1042-6.

2. Roth GA, Johnson C, Abajobir A, Abd-Allah F, Abera SF, Abyu G, et al. Global, Regional, and National Burden of Cardiovascular Diseases for 10 Causes, 1990 to 2015. J Am Coll Cardiol. 2017;70(1):1-25.

\section{Conclusões}

Concluindo, foi identificada uma frequência de diagnóstico de FFA de 2,5\% na avaliação de linha de base do ELSA-Brasil. Idade, sexo masculino, renda, e diagnósticos de doença arterial periférica, insuficiência cardíaca e febre reumática foram associados à FFA. Aproximadamente $90 \%$ da subamostra dos participantes com FFA e uma pontuação $\mathrm{CHA}_{2} \mathrm{DS}_{2}-\mathrm{VASc} \geq 2$ (50\% considerando apenas os diagnosticados por registros de eletrocardiograma) não foram tratados com anticoagulantes.

\section{Contribuição dos autores}

Concepção e desenho da pesquisa: Santos IS, Lotufo P A, Barreto SM, Ribeiro AL, Bensenor IM; Obtenção de dados: Lotufo PA, Brant L, Pinto-Filho MM, Barreto SM, Ribeiro AL, Bensenor I M; Análise e interpretação dos dados: Santos I S, Lotufo P A, Brant L, Pinto-Filho MM, Pereira AC, Barreto SM, Ribeiro AL, Thomas GN, Lip GYH, Bensenor I M; Análise estatística: Santos IS; Obtenção de financiamento: Lotufo PA, Barreto SM, Ribeiro AL, Bensenor I M; Redação do manuscrito: Santos IS, Bensenor I M; Revisão crítica do manuscrito quanto ao conteúdo intelectual importante: Lotufo PA, Brant L, Pinto-Filho MM, Pereira AC, Barreto SM, Ribeiro AL, Thomas GN, Lip GYH.

\section{Potencial conflito de interesse}

Não há conflito com o presente artigo

\section{Fontes de financiamento}

O presente estudo foi financiado pelo Ministério da Saúde do Brasil (Ciência e Departamento de Tecnologia) e o Ministério da Ciência e Tecnologia do Brasil (Financiadora de Estudos e Projetos e Conselho Nacional de Pesquisa do CNPq) (bolsas 01 06 0010,00 RS, 0106 0212,00 BA, 0106 0300,00 ES, 0106 0278,00 MG, 0106 0115,00 SP, 0106 0071,00 RJ).

\section{Vinculação acadêmica}

Não há vinculação deste estudo a programas de pósgraduação.

\section{Aprovação ética e consentimento informado}

Este estudo foi aprovado pelo Comitê de Ética do Hospital Universitário da USP sob o número de protocolo 659/06. Todos os procedimentos envolvidos nesse estudo estão de acordo com a Declaração de Helsinki de 1975, atualizada em 2013. O consentimento informado foi obtido de todos os participantes incluídos no estudo.
3. Kawabata-Yoshihara LA, Scazufca M, Santos IS, Whitaker A, Kawabata VS, Benseñor IM, et al. Atrial fibrillation and dementia: results from the Sao Paulo ageing \& health study. Arq Bras Cardiol. 2012;99(6):1108-14.

4. Marcolino MS, Palhares DM, Benjamin EJ, Ribeiro AL. Atrial fibrillation: prevalence in a large database of primary care patients in Brazil. Europace. 2015;17(12):1787-90. 
5. Benjamin EJ, Levy D, Vaziri SM, D'Agostino RB, Belanger AJ, Wolf PA. Independent risk factors for atrial fibrillation in a population-based cohort. The Framingham Heart Study. JAMA. 1994;271(11):840-4.

6. Norby FL, Soliman EZ, Chen LY, Bengtson LG, Loehr LR, Agarwal SK, et al. Trajectories of cardiovascular risk factors and incidence of atrial fibrillation over a 25-year follow-up: the ARIC Study (Atherosclerosis Risk in Communities). Circulation. 2016;134(8):599-610.

7. Schnabel RB, Sullivan LM, Levy D, Pencina MJ, Massaro JM, D'Agostino RB Sr, et al. Development of a risk score for atrial fibrillation (Framingham Heart Study): a community-based cohort study. Lancet. 2009;373(9665):739-45.

8. Lip G, Freedman B, De Caterina R, Potpara TS. Stroke prevention in atrial fibrillation: past, present and future. Comparing the guidelines and practical decision-making. Thromb Haemost. 2017;117(7):1230-9.

9. Lip GYH, Banerjee A, Boriani G, Chiang CE, Fargo R, Freedman B, et al. Antithrombotic therapy for atrial fibrillation: CHEST Guideline and Expert Panel Report. Chest. 2018;154(5):1121-201.

10. Lane DA, Lip GY. Use of the CHA(2)DS(2)-VASc and HAS-BLED scores to aid decision making for thromboprophylaxis in nonvalvular atrial fibrillation. Circulation. 2012;126(7):860-5.

11. Lip GY, Nieuwlaat R, Pisters R, Lane DA, Crijns HJ. Refining clinical risk stratification for predicting stroke and thromboembolism in atrial fibrillation using a novel risk factor-based approach: the euro heart survey on atrial fibrillation. Chest. 2010;137(2):263-72.

12. Healey JS, Oldgren J, Ezekowitz M, Zhu J, Pais P, Wang J, et al. Occurrence of death and stroke in patients in 47 countries 1 year after presenting with atrial fibrillation: a cohort study. Lancet. 2016;388(10050):1161-9.

13. Lotufo PA. Stroke prevention within primary care: management of atrial fibrillation using oral anticoagulation. Sao Paulo Med J. 2018;136(4):273-5.

14. Nakayama T, Yokoyama T, Yoshiike N, Zaman MM, Date C, Tanaka H, et al. Population attributable fraction of stroke incidence in middle-aged and elderly people: contributions of hypertension, smoking and atrial fibrillation. Neuroepidemiology. 2000;19(4):217-26.

15. Dulli DA, Stanko H, Levine RL. Atrial fibrillation is associated with severe acute ischemic stroke. Neuroepidemiology. 2003;22(2):118-23.

16. Iwahana H, Ishikawa S, Ishikawa J, Kabutoya T, Kayaba K, Gotoh T, et al. Atrial fibrillation is a major risk factor for stroke, especially in women: the Jichi Medical School cohort study. J Epidemiol. 2011;21(2):95-101.

17. Lotufo PA, Goulart AC, Passos VMA, Satake FM, Souza MFM, França EB, et al. Cerebrovascular disease in Brazil from 1990 to 2015: Global Burden of Disease 2015. Rev Bras Epidemiol. 2017 May;20(Suppl 01):129-41.

18. Lotufo PA. Stroke is still a neglected disease in Brazil. Sao Paulo Med J. 2015;133(6):457-9.

19. Aquino EM, Barreto SM, Bensenor IM, Carvalho MS, Chor D, Duncan BB, et al. Brazilian Longitudinal Study of Adult Health (ELSA-Brasil): objectives and design. Am J Epidemiol. 2012;175(4):315-24

20. Schmidt MI, Duncan BB, Mill JG, Lotufo PA, Chor D, Barreto SM, et al. Cohort profile: longitudinal study of adult health (ELSA-Brasil). Int J Epidemiol. 2015;44(1):68-75

21. Pinto MM, Brant LCC, Padilha-da-Silva JL, Foppa M, Lotufo PA, Mill JG, et al. Electrocardiographic findings in brazilian adults without heart disease: ELSABrasil. Arq Bras Cardiol. 2017;109(5):416-24.

22. Macfarlane PW. Evolution of the Glasgow program for computer-assisted reporting of electrocardiograms--1964/1998. Acta Cardiol. 1998;53(2):117-20.
23. Mill JG, Pinto K, Griep RH, Goulart A, Foppa M, Lotufo PA, et al. [Medical assessments and measurements in ELSA-Brasil]. Rev Saude Publica. 2013;47(Suppl 2):54-62.

24. Miname M, Bensenor IM, Lotufo PA. Different methods of calculating ankle-brachial index in mid-elderly men and women: the Brazilian Longitudinal Study of Adult Health (ELSA-Brasil). Braz J Med Biol Res. 2016:49(12):e5734.

25. Verberk WJ, Kollias A, Stergiou GS. Automated oscillometric determination of the ankle-brachial index: a systematic review and meta-analysis. Hypertens Res. 2012;35(9):883-91.

26. Chor D, Alves MG, Giatti L, Cade NV, Nunes MA, Molina MC, et al. Questionnaire development in ELSA-Brasil: challenges of a multidimensional instrument. Rev Saude Publica. 2013;47(Suppl 2):27-36.

27. Goff DC, Lloyd-Jones DM, Bennett G, Coady S, D'Agostino RB, Gibbons R, et al. 2013 ACC/AHA guideline on the assessment of cardiovascular risk: a report of the American College of Cardiology/American Heart Association Task Force on Practice Guidelines. Circulation. 2014;129(25 Suppl 2):S49-73

28. Rahman F, Kwan GF, Benjamin EJ. Global epidemiology of atrial fibrillation. Nat Rev Cardiol. 2014;11(11):639-54.

29. Olesen JB, Lip GY, Lane DA, Køber L, Hansen ML, Karasoy D, et al. Vascular disease and stroke risk in atrial fibrillation: a nationwide cohort study. Am J Med. 2012;125(8):826.e13-23.

30. Brant LCC, Nascimento BR, Passos VMA, Duncan BB, Benseñor IJM, Malta DC, et al. Variations and particularities in cardiovascular disease mortality in Brazil and Brazilian states in 1990 and 2015: estimates from the Global Burden of Disease. Rev Bras Epidemiol. 2017;20(Suppl 01):116-28.

31. Watkins DA, Johnson CO, Colquhoun SM, Karthikeyan G, Beaton A, Bukhman G, et al. Global, Regional, and National Burden of Rheumatic Heart Disease, 1990-2015. N Engl J Med. 2017;377(8):713-22.

32. Ogilvie IM, Newton N, Welner SA, Cowell W, Lip GY. Underuse of oral anticoagulants in atrial fibrillation: a systematic review. Am J Med. 2010;123(7):638-45.e4.

33. Thompson LE, Maddox TM, Lei L, Grunwald GK, Bradley SM, Peterson PN et al. Sex differences in the use of oral anticoagulants for atrial fibrillation: a Report from the National Cardiovascular Data Registry (NCDR) PINNACLE Registry. J Am Heart Assoc. 2017;6(7):e005801.

34. Khurshid S, Weng LC, Hulme OL, Ellinor PT, Lubitz SA. Factors associated with anticoagulation delay following new-onset atrial fibrillation. Am J Cardiol. 2017;120(8):1316-21.

35. Emdin CA, Wong CX, Hsiao AJ, Altman DG, Peters SA, Woodward M, et al Atrial fibrillation as risk factor for cardiovascular disease and death in women compared with men: systematic review and meta-analysis of cohort studies. BMJ. 2016 Jan 19;532:h7013.

36. Alotaibi GS, Almodaimegh H, McMurtry MS, Wu C. Do women bleed more than men when prescribed novel oral anticoagulants for venous thromboembolism? a sex-based meta-analysis. Thromb Res. 2013;132(2):185-9

37. Wolbrette DL. Risk of proarrhythmia with class III antiarrhythmic agents: sex-based differences and other issues. Am J Cardiol. 2003;91(6A):39D44D.

38. Sullivan RM, Zhang J, Zamba G, Lip GY, Olshansky B. Relation of gender-specific risk of ischemic stroke in patients with atrial fibrillation to differences in warfarin anticoagulation control (from AFFIRM). Am J Cardiol. 2012;110(12):1799-802.

\section{* Material suplementar \\ Para informação adicional, por favor, clique aqui.}

